\title{
The silent impact of hearing loss: using longitudinal data to explore the effects on depression and social activity restriction among older people
}

\author{
CLÁUDIA CAMPOS ANDRADE*, CÍCERO ROBERTO PEREIRA† \\ and PEDRO ALCÂNTARA DA SILVA
}

\begin{abstract}
Hearing loss is frequent in old age and has been associated with fewer social activities and depression. However, hearing problems have also been associated with other comorbidities, which prevent more definitive conclusions about the unique role on older people's wellbeing. Moreover, little attention has been paid to the psychological processes through which this relationship occurs. This study aims to investigate the effect of hearing loss on older adults' wellbeing from a longitudinal perspective. Using data from three points in time, we investigated the mutual relationship between hearing loss, depression and social activities. Based on longitudinal data of the Survey of Health, Ageing and Retirement in Europe (SHARE) from ten European countries, we conducted the test of competing auto-regressive crosslagged theoretical models. Results show that hearing loss reduces social activity, which is mediated by depression. The adequacy of this model (versus a model proposing that social activity restriction mediates the relationship between hearing loss and depression) was supported in each of the countries of the sample. Findings showing that hearing loss can contribute to depression and, subsequently, to restriction in social activities have implications for early detection and clinical interventions on hearing loss.
\end{abstract}

KEY WORDS - hearing loss, depression, social activities, longitudinal analysis.

\section{Introduction}

Age-related hearing loss is the most common sensory deficit in the elderly, affecting approximately 20 per cent of individuals over $5^{\circ}$ years of age (Viljanen et al. 2014). With changing population demographics in the developed world, the societal impact of hearing loss is likely to increase in coming

* Instituto Universitário de Lisboa (ISCTE-IUL), CIS-IUL, Lisbon, Portugal.

$\dagger$ Universidade Federal da Paraíba, João Pessoa, Brazil.

† Instituto de Ciências Sociais da Universidade de Lisboa, Lisbon, Portugal. 
years (Carson 2005; Ciorba et al. 2012). Hearing difficulties hinder access to environmental information and may also become an obstacle to communication and social activity, jeopardising independence and overall wellbeing (Heine and Browning 2004; Viljanen et al. 2014). However, literature on the effects of hearing loss is still scarce, and studies on the relationship between hearing loss and depression and between hearing loss and social activity have shown inconsistent results. Those inconsistencies may be due to different evaluation methods and to different study population characteristics (Harada et al. 2008; Wallhagen et al. 2001). Moreover, research has paid little attention to the psychological processes underlying the impact of this sensory difficulty.

A better understanding of how hearing loss influences functioning and wellbeing may enhance our ability to develop creative approaches to minimise its impact (Wallhagen et al. 2001). Based on longitudinal data from the Survey of Health, Ageing and Retirement in Europe (SHARE), the research presented in this paper was undertaken to investigate how hearing loss difficulties are related to depression and social activities using a sample of ten countries. Specifically, we tested if one way through which perceived hearing loss is associated with depression is through social activity restriction. In other words, we examined whether social inactivity mediates the relationship between hearing loss and depression. In addition, we compared this hypothetical model with another one proposing the opposite effect: that people with hearing loss will be less socially active because they have developed more depression.

\section{Effects of hearing loss on depression and social activity}

During the psychological adjustment to hearing loss, people are at risk of psychological problems such as depression (Chen et al. 2013). Varying degrees of depression are commonly associated with living with age-related hearing loss (for a literature review, see Millán-Calenti et al. 2011), but some studies show no such effect. For example, Carabellese et al. (1993) assessed 1,191 non-institutionalised elders aged $7 \mathrm{O}^{-} 75$ years and found that hearing impairment was associated with increased risk of depression. Although not significant, Crews and Campbell (2004) also found that those with hearing loss reported slightly higher rates of depression $(8.0 \%$ versus $5.8 \%)$ than did people without sensory loss. However, cross-sectional designs are unlikely to provide information about the direction of the relationships between hearing loss and depression symptoms. In order to overcome this shortcoming in the studies on the hearing loss effect, Wallhagen et al. (2001) used a longitudinal design and measured hearing loss at one point in time and mental health one year 


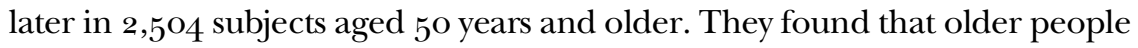
with moderate or more hearing impairment were more likely to feel depressed and to report fair or poor mental health. In another study, Chou (2008) examined the relationship between dual sensory loss and depression in older adults. Results showed that hearing loss only was not a predictor for the onset and persistence of depression. Still, this study evaluated individuals at only two time-points, 24 months apart, which is not enough to shed light on whether the hearing loss effect on depression occurred throughout an indirect pathway. Recently, a 13-year-long study has shown longitudinal relationships between functional health (e.g. hearing impairment) and subsequent levels of positive affect, as an affective dimension of subjective wellbeing, in older people (Gana et al. 2016).

Hearing loss has been characteristically associated with disrupted interaction with others. Patients seeking a hearing aid for the first time were sent an open-ended questionnaire asking them to 'list the difficulties which [they] have as a result of hearing loss' (Stephens 1980: 207). The three most commonly reported problems were difficulties in hearing the television and/or radio, general conversation and hearing the doorbell. From these, difficulties in general conversation were consistently rated as being of greater importance than other problems. Similarly, Gussekloo et al. (2003) found that 40 per cent of older people with untreated severe hearing loss had difficulties in following a group conversation, and that the social isolation caused by this disability was mentioned as the most important reason for obtaining a hearing aid in the future, especially the possibility of having difficulties in communicating with loved ones, such as children and grandchildren.

Helvik, Jacobsen and Hallberg (2006) describe the documented consequences of hearing loss in terms of perceived activity limitation and participation restriction. Negative auditory consequences are difficulties such as determining the location of sound, in perception of speech, in discriminating speech from background sound and in hearing non-verbal sounds, which may be expressed as activity limitations. Moreover, negative nonauditory consequences are, for instance, social withdrawal, reduced participation in social activities outside the family and limited prospects of promotion in employment. Such consequences refer to difficulties in engagement in everyday life situations and might be classified as participation restrictions. These authors found that the perceived severity of hearing loss predicted both activity limitation and participation restriction, which is consistent with further evidence showing that hearing impairment has an impact on social functioning, with persons reporting even a mild hearing impairment being more likely to feel lonely, remote or left out, and have more difficulty paying attention (Wallhagen et al. 2001). 
More specifically, older people with hearing loss have reported greater difficulties with activities and social roles than did people without sensory problems (Crews and Campbell 2004). They were as likely as those without sensory impairment to eat out at a restaurant, get exercise and visit relatives, but less likely to visit friends, phone friends, phone relatives, attend church and go to movies. About one-quarter (25.1\%) of older people with hearing loss reported having too little social activity, and that they would like to do more, compared to one-fifth (21.6\%) of older people without sensory loss. Based on the 2004 data of the SHARE, comprising 27,536 men and women aged 50 years and older, Viljanen $e t$ al. (2014) confirmed that participants who reported hearing difficulties had 1.23 $(95 \%$ confidence interval $(\mathrm{CI})=1.10-1.37)$ times higher unadjusted odds for social inactivity compared to persons without hearing or vision difficulties.

Given the importance of social contacts and relationships to wellbeing in old age (Cornwell and Waite 2009), the consequences of hearing loss are cause for concern. However, there have also been incongruent findings. Studies have found that hearing loss was associated with increased risk of poor functioning in daily living activities, but not with fewer social relationships (Carabellese et al. 1993; Yamada et al. 2012). In the same way, Harada et al. (2008) found no relationship between hearing loss and reduced functional activity (i.e. instrumental self-maintenance, intellectual and social role activities).

Again, these studies report cross-sectional data in that no information about the direction of the causal chain is provided. But there is an additional factor preventing us from more definitive conclusions about the specific role of hearing loss, which is the confounding relation between hearing loss and poorer physical functioning in older adults (e.g. Bess, Lichtenstein and Logan 1990; Bess et al. 1989; Chen et al. 2014). For example, Crews and Campbell (2004) found that people with hearing loss were more likely to report difficulty walking, getting outside, getting into and out of a bed or chair, and managing medication, than were people without sensory problems. Moreover, those with hearing loss were also more likely to have experienced falls, to report heart disease and to report stroke, as well as less likely to perceive health as excellent or very good. A number of studies have also shown a correlation between hearing loss and cognitive decline (for a review, see Arlinger 2003). Taken together, these results show that people with hearing loss report higher rates of comorbid and secondary conditions than did people who did not have any sensory loss, indicating that hearing loss might occur in the context of other age-related physiological and psycho-social changes (see also Dalton et al. 2003). 
For example, Chou and Chi (2004) found that hearing loss was significantly associated with depression, but the inclusion of self-rated health made this relationship diminish to an insignificant level. In another study, adjusting for physical disability, the association between hearing difficulty and depression was still consistent but not significant (Jones, Victor and Vetter 1984). Research has shown that perceived hearing loss as measured by self-report has an impact on depression, either through cross-sectional studies, or through studies that collect data at two points in time. However, this body of evidence does not elucidate the direction of the relationship, nor how that process relates to the decrease in social activities.

\section{How does hearing loss affect depression and social activities? The importance of the temporal order}

To our knowledge, no studies have addressed the psychological processes through which hearing loss may affect depression and frequency of social activities. It is plausible that hearing loss, being the cause of reduced communicative relationships, as well as reduced social and emotional interactions, would be a source of loneliness, isolation and depression (Millán-Calenti et al. 2011). As some studies have suggested, hearing impairments can disrupt interpersonal relations and limit participation in desired roles, both of which would negatively affect the emotional wellbeing of older persons (Wallhagen et al. 2001). For example, Hallberg, Påsse and Jansson (1999) examined the impact of selfreported disability and handicap on psychological general wellbeing among women with noise-induced hearing loss. They found that interpersonal distress (i.e. feeling restrictions in social life, being tense and tired, lack of self-confidence, feelings of being cut off, being avoided by other people and impact of hearing loss on close relationships) was the strongest predictor, and that difficulties in hearing speech had also a negative impact on the sense of general wellbeing.

There is a long research history linking social relationships and health. Social support is a valuable and effective means by which a person can reduce the effects of stressful experiences, and greater social integration is associated with better psychological wellbeing and more positive affective states (Cohen 2004). If people with hearing loss find it harder to benefit from their usual sources of social support, or disengage from social roles and relationships, increasing levels of isolation will have a negative impact on health. Moreover, meaningful activities and social integration are associated with positive outcomes in later life and are prerequisites for successful ageing (Silverstein and Parker 2002). Thus, social inactivity may have considerable negative psychological effects, particularly when withdrawal from activities due to sensory impairment is involuntary. 
Another possibility is that hearing loss may first cause depression, which in turn may lead to a decrease in social activities (e.g. Wilkie et al. 2007). Research suggests that depression is often a response to declining health and functional impairment in the older adult. On the other hand, from a psychopathological perspective, a decreased interest or pleasure in all or most activities is a symptom of depression (American Psychiatric Association 2013), which means that depression as a major disorder can diminish individual's motivation to enroll in action, and engage in social activities. Accordingly, hearing loss could contribute to worsen a depressive disorder, which consequently would lead to a reduced involvement in activities.

Overall, research suggests that hearing loss consequences in terms of emotional wellbeing and social engagement are complex, and most likely multidirectional. Depression can negatively affect social relationships, but eventually a cycle of increasingly restricted activities may limit social contacts, which may also contribute to isolation and poor mental health (Viljanen et al. 2014). Nevertheless, interventions to prevent hearing-loss negative consequences from the onset may be critical and, for that, the ability to identify the specific effect of hearing loss on depression and social activity and the causal sequencing involved in this psychological process is paramount. Therefore, this study examines different possibilities: that hearing loss may cause depression because it leads to less social activity, that hearing loss may cause less social activity because it leads to depression or that the effects are reciprocal and all variables mutually influence each other.

\section{Method}

\section{Participants}

This study is based on the 2004 (Wave 1), 2006 (Wave 2) and 2010 (Wave 4) data collection of the SHARE (Wave 3, SHARELIFE, was not included because it focused on people's life histories). Waves 1, 2 and 4 comprised

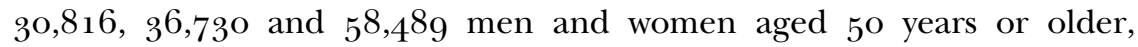
respectively.

SHARE includes a great variety of information about health, socio-economics and social networks, and is based on 2004, 2006 and 2010 representative samples from 11 European countries (Sweden, Denmark, Germany, the Netherlands, Belgium, France, Switzerland, Austria, Italy, Spain and Greece). Three countries joined SHARE in 2006-Czech Republic, Poland and Ireland - and four countries joined SHARE in 2010-Estonia, Hungary, Portugal and Slovenia. Because the present study is based on longitudinal data at three time-points, we only used data from ten countries, i.e. those that have contributed data to the 2004 SHARE baseline study and that 
have remained in Waves 3 and 4 (Greece was not part of Wave 4 ). From these ten countries, of the 30,816 participants in Wave 1, 10,697 form a longitudinal sample who answered to the three waves. Thus, in the final sample there were $5,681(56.3 \%)$ women and $4,407(43.7 \%)$ men, who at Wave 4 (2010) had an average age of 69 years (standard deviation $(\mathrm{SD})=9.02$ years). Descriptive analysis showed that participants increased the use of a hearing aid from one wave to another, which indicates an increase of hearing loss with age (for more characteristics of the sample, see Table 1 ).

\section{Procedure}

Data were collected using computer-assisted personal interviews by trained interviewers at participants' homes (Jürges 2005). Ethical approval for the SHARE was obtained from the University of Mannheim's internal review board, Germany, and all the participants gave their informed consent before the interview (Börsch-Supan and Jürges 2005).

\section{Measures}

Several comparative studies support the validity of self-reported data of sensory impairment (Wallhagen et al. 2001). Hearing loss was assessed with the question 'Is your hearing (using a hearing aid as usual) excellent/very good/good/fair/poor?' (from 1 'excellent' to 5 'poor'). To measure depressive symptoms, the Euro-depression scale (EURO-D; Prince et al. 1999) was used. The EURO-D scale is the number of depressive symptoms reported by a person and comprises the following 12 items: depression, pessimism, suicidality (death wish), guilt, sleep, interest, irritability, appetite, fatigue, concentration, enjoyment and tearfulness, with higher numbers reflecting more depression symptoms. Finally, the frequency of social activity was assessed through six items: 'Done voluntary or charity work'; 'Attended an educational or training course'; 'Gone to a sport, social or other kind of club'; 'Taken part in activities of a religious organisation'; 'Taken part in a political or community-related organisation'; and 'Look after children [grandchild/grandchildren] without the presence of the parents', which were common across the three waves. The response categories on the rating scale were standardised to: 'never' (o), 'less often than every week' (1), 'every week' (2), 'daily' (3).

\section{Statistical analyses}

First, we excluded the individuals who had not answered one of the variables in all the three waves included in the analyses. This resulted in a final sample 
TA B LE 1. Participants' characteristics

\begin{tabular}{lr}
\hline & $\mathrm{N}(\%)$ \\
\hline Country: & \\
Austria & $583(5.8)$ \\
Germany & $876(8.7)$ \\
Sweden & $1,143(11.3)$ \\
The Netherlands & $1,085(10.8)$ \\
Spain & $850(8.4)$ \\
Italy & $1,225(12.1)$ \\
France & $1,162(11.5)$ \\
Denmark & $818(8.1)$ \\
Switzerland & $477(4.7)$ \\
Belgium & $1,869(18.5)$ \\
Use a hearing aid: & \\
Time 1 (Wave 1, 2004) & $392(3.9)$ \\
Time 2 (Wave 2, 2006) & $491(4.9)$ \\
Time 3 (Wave 4, 2010) & $803(8.0)$ \\
\hline
\end{tabular}

of 10,088 individuals. To analyse longitudinal mean changes, we conducted structural equation modelling with latent variable to estimate Latent Growth Curve Analysis (Duncan, Duncan and Stryker 2006). Then a wellsuited statistical approach for the longitudinal analysis of alternative causal models was performed - the auto-regressive cross-lagged analysis (Finkel 1995). The auto-regressive cross-lagged analysis is widely used to shed light on the nature of the causal associations between variables in non-experimental, longitudinal research designs (Selig and Little 2012). In each analysis, hearing loss, depression and social activity were specified as continuous variables.

First we estimated a stability model (Model $1\left(M_{1}\right)$ ), which includes the auto-regressive effects between time 1 ( $\mathrm{T}_{1}$, Wave 1 ), time 2 ( $\mathrm{T}_{2}$, Wave 2 ) and time $3\left(\mathrm{~T}_{3}\right.$, Wave 4$)$ for each variable, as well as the correlations between the variables at $T_{1}$ (see online Supplementary Figure 1). In this model, and in the subsequent models, each of the variables at $\mathrm{T}_{1}-$ hearing loss, social activities and depression - were linked to the same variables at $\mathrm{T}_{2}$ and $\mathrm{T}_{3}$. Secondly, the model representing the hypothesis that hearing loss produces depression through a reduction in social activities (M2) was tested, in which the direct causality relations were added to M1 (see Figure 1). Then we estimated the reverse causality model $\left(\mathrm{M}_{3}\right)$, proposing the reverse effects. In other words, depression at $\mathrm{T}_{1}$ predicts social activities at $\mathrm{T}_{2}$, and social activities at $\mathrm{T}_{2}$ predict hearing loss at $\mathrm{T}_{3}$ (see online Supplementary Figure 2). $\mathrm{M}_{4}$ represents the hypothesis that hearing loss leads to reduction in social activities through depression (see Figure 2), and $\mathrm{M}_{5}$ tested the reverse effects, with social activities at $\mathrm{T}_{1}$ predicting depression at $\mathrm{T}_{2}$, and depression at $\mathrm{T}_{2}$ predicting hearing loss at $\mathrm{T}_{3}$ (see 


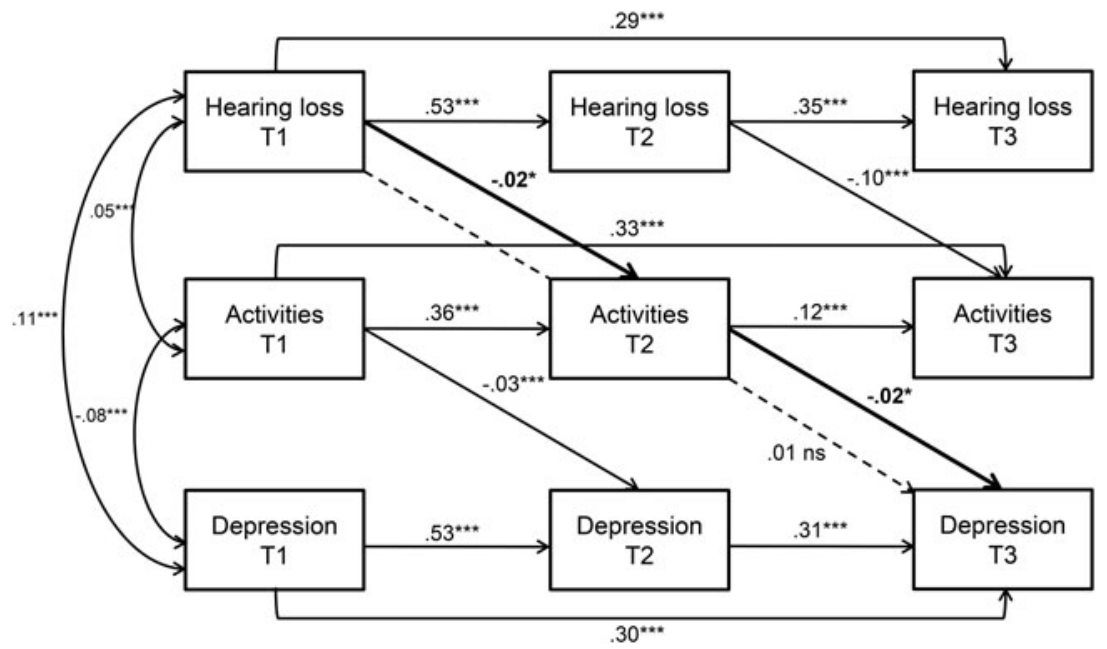

Figure 1. Estimated parameters of the model proposing activities as the mediator between hearing loss and depression (Model 2). T1: time 1 (Wave 1, 2004). T2: time 2 (Wave 2, 2006). $\mathrm{T}_{3}$ : time 3 (Wave 4, 2010). Curved arrows represent correlation effects; solid straight arrows represent direct effects; dashed straight arrow represents direct effect controlling for the effect of the mediator.

Significance levels: $* p<0.05, * * * p<0.001$, ns: not significant.

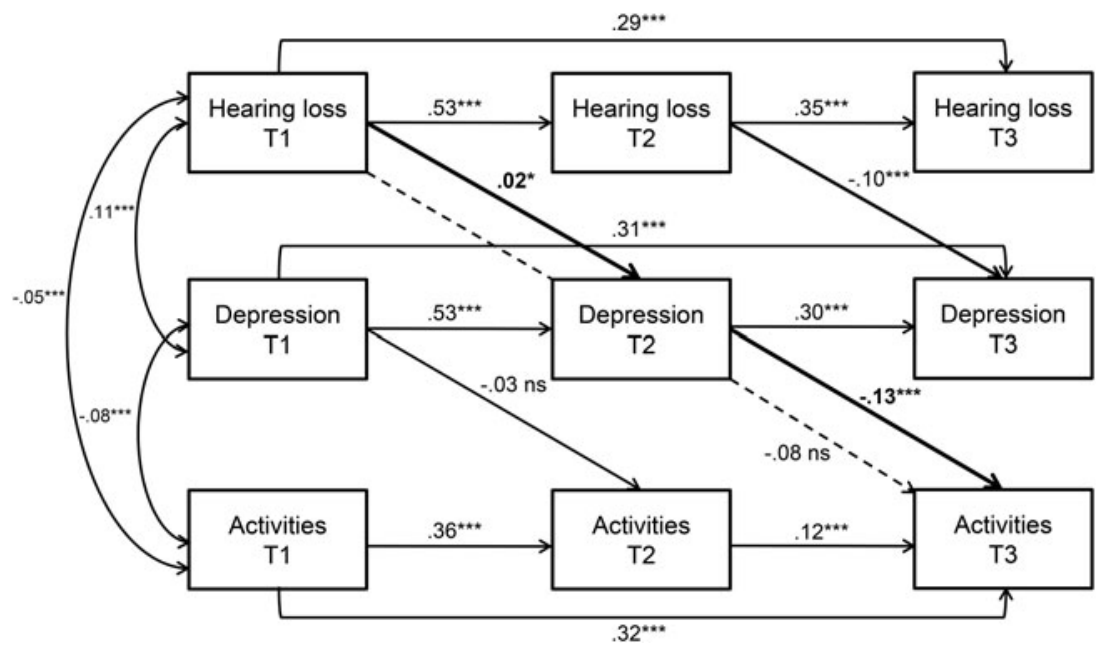

Figure 2. Estimated parameters of the model proposing depression as the mediator between hearing loss and activities (Model 4). T1: time 1 (Wave 1, 2004). T2: time 2 (Wave 2, 2006). T3: time 3 (Wave 4, 2010). Curved arrows represent correlation effects; solid straight arrows represent direct effects; dashed straight arrow represents direct effect controlling for the effect of the mediator.

Significance levels: $* p<0.05$, $* * * p<0.001$, ns: not significant. 
online Supplementary Figure 3). Finally, we estimated a reciprocal causality model (M6), which combines $\mathrm{M}_{1}, \mathrm{M}_{2}, \mathrm{M}_{3}, \mathrm{M}_{4}$ and $\mathrm{M}_{5}$ (see Figure 3). This model allows assessment all together of the various possibilities by which perceived hearing loss has an impact on depression and practice of social activities and is mutually influenced by these.

To evaluate the global adjustment of the models, we considered the Comparative Fit Index (CFI) and Goodness-of-Fit Index (GFI) above o.9o, and the Root Mean Square Error of Approximation (RMSEA) below 0.05 as indicating a good fit of the model to the data (e.g. Schumacker and Lomax 1996). In addition, we used the chi-square difference test and the Bayesian Information Criterion (BIC) to compare the goodness of fit of the models. The best models are those with lower chi-square and BIC values (Byrne 2009).

\section{Results}

\section{Preliminary analyses}

Tables 2 and 3 present, respectively, the descriptive statistics and the correlation matrix for the model variables. We started by conducting a dropout analysis comparing the longitudinal sample $(N=10,088)$ to the participants who were interviewed at T1 but did not participate in all three waves $(N=$ $20,728)$. We found differences in the three variables. Individuals who did not participate in the three rounds exhibited higher scores in both hearing loss $($ mean $=2.85, \mathrm{SD}=1.06$ versus mean $=2.54, \mathrm{SD}=1.05 ; t=$ $7.08, p<0.001)$ and depression $\left(\right.$ mean $=2.92, \mathrm{SD}=2.5^{\circ}$ versus mean $=$ $2.20, \mathrm{SD}=2.12 ; t=7.34, p<0.001)$, and showed lower scores of social activities $($ mean $=0.22, \mathrm{SD}=0.35$ versus mean $=0.31, \mathrm{SD}=0.34 ; t=-6.11$, $p<0.001)$. This pattern of results suggests that depression is associated with less involvement in social activities and more hearing loss, and that the dropout sample apparently had lower psychological and functional health.

To compare the two samples further, and of the most importance for our proposal, we inspected the correlation matrices between the three variables at $T_{1}, T_{2}$ and $T_{3}$. These results showed that, in both samples, the correlations between hearing loss and frequency of social activities are negative (meaning that the greater the hearing loss, the lower the frequency of social activities), that the correlations between hearing loss and level of depression are weak and positive (indicating that the greater the hearing loss, the higher the level of depression), and that the correlations between social activities and depression are weak and negative (meaning that the higher the frequency of activities, the lower the level of depression). 


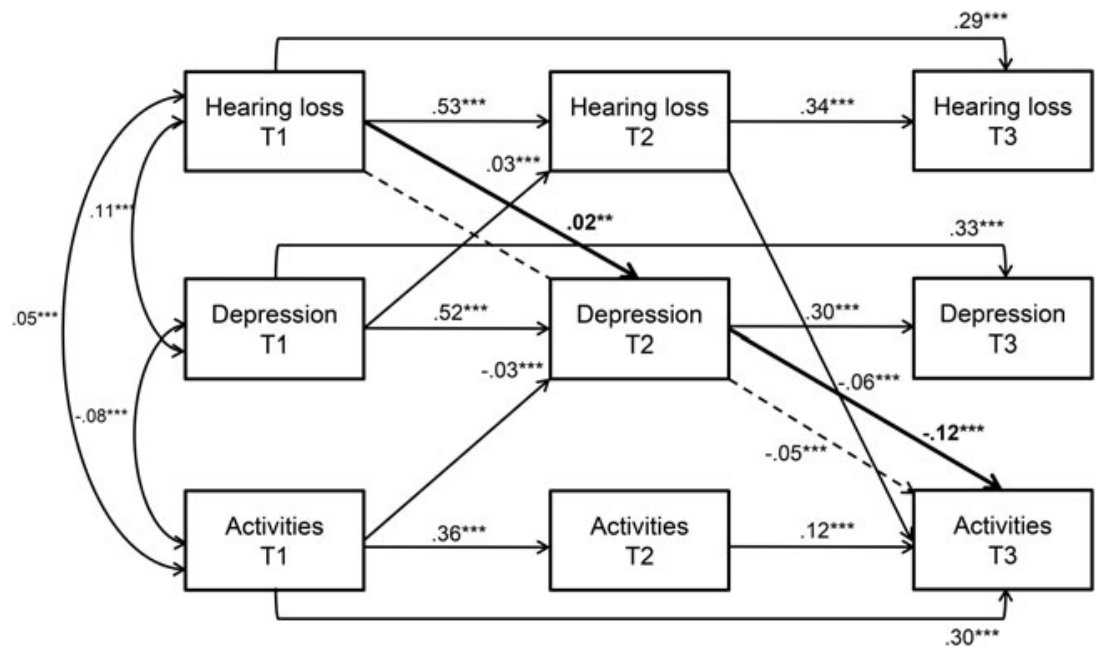

Figure 3. Estimated parameters of the reciprocal causality model (Model 6). T1: time 1 (Wave 1, 2004). T2: time 2 (Wave 2, 2006). T3: time 3 (Wave 4, 2010). Curved arrows represent correlation effects; solid straight arrows represent direct effects; dashed straight arrow represents direct effect controlling for the effect of the mediator.

Significance levels: $* p<0.05, * * p<0.01, * * * p<0.001$

T A B L E 2. Means and standard deviations of the variables at each point in time, and estimated parameters from the Latent Growth Curve Analyses

\begin{tabular}{llcc}
\hline & Hearing loss & Social activities & Depression \\
\hline Means (standard deviations) & & & \\
Time 1 (Wave 1, 2004) & $2.54(1.05)$ & $0.31(0.34)$ & $2.20(2.12)$ \\
Time 2 (Wave 2, 2006) & $2.62(1.02)$ & $0.3^{\circ}(0.36)$ & $2.14(2.09)$ \\
Time 3 (Wave 4, 2010) & $2.72(1.03)$ & $0.7^{\circ}(0.43)$ & $2.37(2.17)$ \\
Latent growth models: & & & \\
Model 1 (linear slope): & & & \\
Intercept & $2.54^{* * *}$ & $0.25^{* * *}$ & $2.16^{* * *}$ \\
Slope & $0.09^{* * *}$ & $0.16^{* * *}$ & $0.08^{* * *}$ \\
CFI & 1.00 & 0.27 & 0.99 \\
RMSEA & 0.00 & 0.49 & 0.08 \\
Model 2 (quadratic added): & & & \\
Intercept & $2.54^{* * *}$ & $0.31^{* * *}$ & $2.20^{* * *}$ \\
Slope & $0.08^{* * *}$ & $-0.21^{* * *}$ & $-0.19^{* * * *}$ \\
Quadratic & 0.01 & $0.20^{* * *}$ & $0.14^{* * *}$ \\
CFI & 1.00 & 1.00 & 1.00 \\
RMSEA & 0.00 & 0.00 & 0.00 \\
& & &
\end{tabular}

Notes: CFI: Comparative Fit Index. RMSEA: Root Mean Square Error of Approximation. Hearing loss: minimum $=1$, maximum $=5$. Social activities: minimum $=0$, maximum $=3$. Depression: minimum $=0$, maximum $=12$.

Significance level: $* * * p<0.001$. 
T A B L E 3. Correlation matrix of the variables at each point in time

\begin{tabular}{|c|c|c|c|c|c|c|c|c|c|}
\hline & $\mathrm{HL}_{1}$ & HL2 & $\mathrm{HL}_{3}$ & $A_{1}$ & $\mathrm{~A}_{2}$ & $\mathrm{~A}_{3}$ & $D_{1}$ & $\mathrm{D}_{2}$ & $\mathrm{D}_{3}$ \\
\hline \multicolumn{10}{|l|}{ Hearing loss: } \\
\hline $\mathrm{T}_{1}(\mathrm{HL} 1)$ & 1 & $0.534^{* * *}$ & $0.477^{* * *}$ & $-0.052^{* * *}$ & $-0.039 * * *$ & $-0.109 * * *$ & $0.108 * * *$ & $0.080^{* * * *}$ & $0.102 * * *$ \\
\hline $\mathrm{T}_{2}$ (HL2) & & 1 & $0.502 * * *$ & $-0.047^{* * *}$ & $-0.035^{* * *}$ & $-0.118 * * *$ & $0.093 * * *$ & $0.119 * * *$ & $0.102 * * *$ \\
\hline $\mathrm{T}_{3}\left(\mathrm{HL}_{3}\right)$ & & & 1 & $-0.050^{* * * *}$ & $-0.046^{* * *}$ & $-0.120^{* * *}$ & $0.079^{* * * *}$ & $0.081 * * *$ & $0.140^{* * *}$ \\
\hline \multicolumn{10}{|c|}{ Social activities: } \\
\hline $\mathrm{T}_{1}\left(\mathrm{~A}_{1}\right)$ & & & & 1 & $0.361 * * *$ & $0.37^{* * * *}$ & $-0.083^{* * *}$ & $-0.075^{* * *}$ & $-0.091 * * *$ \\
\hline $\mathrm{T}_{2}\left(\mathrm{~A}_{2}\right)$ & & & & & 1 & $0.243^{* * *}$ & $-0.045^{* * *}$ & $-0.048^{* * *}$ & $-0.047^{* * * *}$ \\
\hline $\mathrm{T}_{3}\left(\mathrm{~A}_{3}\right)$ & & & & & & 1 & $-0.166 * * *$ & $-0.161 * * *$ & $-0.210^{* * *}$ \\
\hline \multicolumn{10}{|l|}{ Depression: } \\
\hline $\mathrm{T}_{1}\left(\mathrm{D}_{1}\right)$ & & & & & & & 1 & $0.530^{* * *}$ & $0.47 \mathrm{O}^{* * *}$ \\
\hline $\mathrm{T}_{2}\left(\mathrm{D}_{2}\right)$ & & & & & & & & 1 & $0.470^{* * * *}$ \\
\hline $\mathrm{T}_{3}\left(\mathrm{D}_{3}\right)$ & & & & & & & & & 1 \\
\hline
\end{tabular}

Notes: T1: time 1 (Wave 1, 2004). T2: time 2 (Wave 2, 2006). T3: time 3 (Wave 4, 2010). HL1, HL2 and HL3: hearing loss at $\mathrm{T}_{1}, \mathrm{~T}_{2}$ and $\mathrm{T}_{3}$, respectively. $\mathrm{A}_{1}, \mathrm{~A}_{2}$ and $\mathrm{A}_{3}$ : activities at $\mathrm{T}_{1}, \mathrm{~T}_{2}$ and $\mathrm{T}_{3}$, respectively. $\mathrm{D}_{1}, \mathrm{D}_{2}$ and $\mathrm{D}_{3}$ : depression at $\mathrm{T}_{1}, \mathrm{~T}_{2}$ and $\mathrm{T}_{3}$, respectively.

Significance level: $* * * p<0.001$ 
This pattern of results also occurred when we analysed the interrelationship between the three variables in the cross-sectional sample of 30,816 individuals from Wave 1 , with all correlations being significant both in the final and in the dropout sample $(p<0.001)$. Thus, this dropout analysis suggests that the longitudinal sample is similar to the total sample at $\mathrm{T} 1$.

Focusing now only on the final sample, we analysed longitudinal mean changes by using Latent Growth Curve Analysis (Duncan, Duncan and Stryker 2006). We specified two models, one assuming a linear trend model and one saturated model predicting a quadratic change. As one can see in Table 2, the linear model fits better for hearing loss, while the quadratic models fit better for depression and social activities changes. Indeed, results show that hearing loss increases linearly from $\mathrm{T}_{1}$ (mean = $2.54, \mathrm{SD}=1.05)$ to $\mathrm{T}_{2} \quad($ mean $=2.62, \mathrm{SD}=1.02)$ and from $\mathrm{T}_{2}$ to $\mathrm{T}_{3}$ $($ mean $=2.72, \mathrm{SD}=1.03)$. Regarding depression, there are also differences over time, but the changing trend follows a quadratic trend in that the level of depression decreased from $\mathrm{T}_{1}($ mean $=2.2 \mathrm{O}, \mathrm{SD}=2.12)$ to $\mathrm{T}_{2}($ mean $=$ 2.14, $\mathrm{SD}=2.09)$, but increased at $\mathrm{T}_{3}($ mean $=2.37, \mathrm{SD}=2.17)$. For the frequency of social activities, the quadratic effect occurred because there were no changes from $\mathrm{T}_{1}($ mean $=0.31, \mathrm{SD}=0.34)$ to $\mathrm{T}_{2}($ mean $=0.3 \mathrm{O}$, $\left.\mathrm{SD}=0.3^{6}\right)$, but it increased at $\mathrm{T}_{3}($ mean $=0.70, \mathrm{SD}=0.43)$.

\section{Cross-lagged analyses}

Table 4 presents the goodness-of-fit for each tested model.

We started by testing the stability model (M1, see online Supplementary Figure 1) which estimates the auto-regressive effects between $\mathrm{T}_{1}, \mathrm{~T}_{2}$ and $\mathrm{T}_{3}$ for each variable. This model fitted very well to the data and it is useful to provide information about the stability of the measures over time and helps to control for third variables that might account for effects predicted by our hypotheses. Stability estimates for hearing loss were $0.5^{\mathrm{O}}$ from $\mathrm{T}_{1}$ to $\mathrm{T}_{2}$, and $0.5^{2}$ from $\mathrm{T}_{2}$ to $\mathrm{T}_{3}$. Stability for social activities was less strong: 0.36 from $\mathrm{T}_{1}$ to $\mathrm{T}_{2}$, and 0.24 from $\mathrm{T}_{2}$ to $\mathrm{T}_{3}$. Stability for depression was strong from $\mathrm{T}_{1}$ to $\mathrm{T}_{2}(0.53)$, and from $\mathrm{T}_{2}$ to $\mathrm{T}_{3}(0.47)$. As could be expected, at $\mathrm{T}_{1}$ there were within-time positive correlations between hearing loss and depression, and a negative association between depression and social activities, as well as between hearing loss and social activities.

M2, proposing the frequency of social activities as mediator between hearing loss and depression, showed a good fit to the data (see Figure 1), that is, significantly better than the fit of the stability model $\left(\Delta \chi^{2}=\right.$ 3043.066, $\Delta$ df (degrees of freedom) $=8, p<0.001)$. Estimated parameters indicated that hearing loss at $\mathrm{T}_{1}$ implies fewer social activities at $\mathrm{T}_{2}$ and fewer social activities at $\mathrm{T}_{2}$ predict more depression symptoms at $\mathrm{T}_{3}$, but 
T А в LE 4. Goodness-of-fit indices of the estimated models

\begin{tabular}{|c|c|c|c|c|c|c|c|c|}
\hline & $\chi^{2}$ & df & CFI & GFI & RMSEA & $p$-close & BIC & $\Delta \chi^{2}($ versus Model 1$)$ \\
\hline Model 1: Stability model & $799.75^{\circ}$ & 24 & 0.957 & 0.982 & 0.057 & 0.001 & $993 \cdot 35^{1}$ & \\
\hline Model 2: Social activities as mediator & 634.046 & 19 & 0.966 & 0.986 & 0.057 & 0.002 & 873. & $165 \cdot 704 * * *$ \\
\hline Model 3: Social activities as mediator - reversed causality & 574.723 & 19 & 0.969 & 0.987 & 0.054 & 0.045 & 814.419 & $252.027^{* * * *}$ \\
\hline Model 4: Depression as mediator & 490.307 & 19 & 0.974 & 0.989 & $0.05^{\circ}$ & $0.5^{62}$ & 730.004 & $309.443^{* * *}$ \\
\hline Model 5: Depression as mediator - reversed causality & 754.122 & 19 & 0.959 & 0.983 & 0.062 & 0.000 & 993.819 & 45.638 *** \\
\hline Model 6: Reciprocal causality model & 390.549 & 8 & 0.979 & 0.991 & 0.069 & 0.000 & 731.655 & $409.201 * * *$ \\
\hline
\end{tabular}

Notes: df: degrees of freedom. CFI: Comparative Fit Index. GFI: Goodness-of-Fit Index. RMSEA: Root Mean Square Error of Approximation. p-close ( $p$-value for the closeness of RMSEA). BIC: Bayesian Information Criterion.

Significance level: $* * * p<0.001$. 
these effect are not reliable at $p<0.01$. Also, this model provides us with initial information on how hearing loss influences depression: there is a direct reliable effect from hearing loss at $\mathrm{T}_{1}$ to depression at $\mathrm{T}_{3}$, but this effect is not due to lower involvement in social activities at $\mathrm{T}_{2}$.

The reverse causality model $\left(\mathrm{M}_{3}\right.$, see online Supplementary Figure 2$)$ also showed a good fit to the data, significantly better than the stability model $\left(\Delta \chi^{2}=3102.389, \Delta \mathrm{df}=8, p<0.001\right)$, and better than $\mathrm{M}_{2}$ according to the BIC estimates. Estimated parameters of $\mathrm{M}_{3}$ showed that depression at $\mathrm{T}_{1}$ did not predict social activity at $\mathrm{T}_{2}$, but more social activities at $\mathrm{T}_{2}$ implies less perceived hearing loss at $\mathrm{T}_{3}$. Estimating this model is important because, apart from being an alternative to $\mathrm{M}_{2}$, it can elucidate about the direction of causality between the target variables. $\mathrm{M}_{3}$ shows that social activities at $\mathrm{T}_{3}$ are predicted by depression at $\mathrm{T}_{2}$, which suggests the possibility that depression is a mediator of the influence of hearing loss in social activities, as tested by $\mathrm{M}_{4}$.

$\mathrm{M}_{4}$ proposes depression as a mediator between hearing loss and reduction in social activities (see Figure 2). Results showed a very good fit to the data, significantly better than the stability model $\left(\Delta \chi^{2}=3186.805, \Delta \mathrm{df}=8\right.$, $p<0.001)$, and better than $\mathrm{M}_{2}$ and $\mathrm{M}_{3}$ according to $\mathrm{BIC}$ estimates. Estimated parameters indicate that hearing loss at $\mathrm{T}_{1}$ implies more depression at $\mathrm{T}_{2}$, and greater depression at $\mathrm{T}_{2}$ leads to less activity at $\mathrm{T}_{3}(p<$ o.001). Importantly, the indirect effect (mediated by depression at T2) was reliable with bootstrapping procedures using 5,ooo bias-corrected resamples (indirect effect $=-0.0010,90 \% \mathrm{CI}=-0.0020$ to -0.0001 ). The fact that $\mathrm{M}_{4}$ fits the data better than $\mathrm{M}_{2}$ provides additional evidence that it is more likely that hearing loss has an impact on social activities through depression, than that it has an effect on depression by means of reducing social activities.

$\mathrm{M}_{5}$ tests another reversed causality process $\left(\mathrm{M}_{5}\right.$, see online Supplementary Figure 3$)$. Results showed that this model fitted well to the data, significantly better than the stability model $\left(\Delta \chi^{2}=2922.99, \Delta \mathrm{df}=8, p<0.001\right)$, but worse than all the others. It is important to estimate this model because it allows verification that it is less likely that the process occurs from social activities reduction to hearing loss through depression than the other way around.

The reciprocal causality model (M6, see Figure 3) fits better to the data than all the previous ones. In terms of reciprocal relations between the three variables, estimated parameters indicate that greater hearing loss at $\mathrm{T}_{1}$ implies more depression at $\mathrm{T}_{2}$, but depression at $\mathrm{T}_{2}$ does not predict more hearing loss at $T_{3}$, which supports the idea that the process starts with hearing loss and not the opposite. On the other hand, hearing loss and social activities show no reciprocal relations between them. Finally, the relationship between depression and social activities is reciprocal: 
more social activities at $\mathrm{T}_{1}$ predict less depression at $\mathrm{T}_{2}$, and more depression at $\mathrm{T}_{2}$ implies less involvement in social activities at $\mathrm{T}_{3}$.

More importantly, M6 allows identification of the processes through which hearing loss at $\mathrm{T}_{1}$ predicts depression and social activity at $\mathrm{T}_{3}$. In fact, previous results had already shown that $\mathrm{M}_{4}$, which proposes depression as mediator, is better than M2, which tests social activities as mediator. Finally, M6 confirms that depression mediates the effect of hearing loss on social activities and that this mediation is reliable with bootstrapping procedures using 5 ,ooo bias-corrected resamples (indirect effect $=-0.015,90 \% \mathrm{CI}=-0.019$ to -0.011 ). In fact, hearing loss at $\mathrm{T}_{1}$ leads to greater depression at T2 $(b=0.02, p=0.01)$, and more depression at $\mathrm{T}_{2}$ leads to less involvement in social activities at $\mathrm{T}_{3}$ ( $b=-0.12, p<0.001)$. Moreover, M6 shows that the path leading from depression at $\mathrm{T}_{2}$ to social activities at $\mathrm{T}_{3}$ is reliable, while the path leading from social activities at $\mathrm{T}_{2}$ to depression at $\mathrm{T}_{3}$ is not. Overall, these results reinforce evidence that the process starts with perceived hearing loss, which has an impact on social activity reduction over time; and that this influence occurs because hearing loss has an effect on depression. Moreover, M6 has very good fit indices when estimated only with male participants $\left(\mathrm{X}^{2}(8)=\right.$ 109.936, $p<0.001 ; \quad \mathrm{CFI}=0.975 ; \quad \mathrm{GFI}=0.990 ; \quad \mathrm{RMSEA}=0.072 ; \quad p$-close $(p$-value for the closeness of RMSEA $)<0.001 ; \mathrm{BIC}=500.402)$ and only with female participants $\left(\mathrm{X}^{2}(8)=269.998, p<0.001 ; \mathrm{CFI}=0.974 ; \mathrm{GFI}=0.989\right.$; RMSEA $=0.076$; $p$-close $<0.001 ; \mathrm{BIC}=589.859)$. This is important because it shows that the processes by which hearing loss predicts social activity, being this effect mediated by depression, is the same for men and women.

\section{Supplementary analyses}

A series of complementary analyses were performed in order to test the adequacy of M6 in each of the countries in the sample (see online Supplementary Table 1). Although the model seems to be less good in Austria and Switzerland, with CFI values of 0.95 and 0.94 , respectively, in general, the model fit is excellent in all countries, with all adjustment indices well above the recommended values. These results are important because they give further empirical support to M6, the reciprocal causality model, as the best model representing the psychological processes linking perceived hearing loss to depression and restrictions in social activities.

\section{Discussion}

Drawing upon cross-national multi-wave panel data from ten different European countries, the purpose of this study was to investigate the 
effects over time of hearing loss on older adults' depression and social engagement from a longitudinal perspective. Using data from three points in time, we investigated the mutual relationship between hearing loss, depression and social activities. For doing so, we examined the empirical adequacy of different theoretical models. These models proposed either that perceived hearing loss predicts fewer social activities, and that activity restriction predicts depression; or that perceived hearing loss predicts depression, which leads to fewer social activities; the reversed causalities; or presumed reciprocal causal relations between all the variables.

Results showed that the reciprocal causality model fits best to the data. This model shows that there are reciprocal relations between depression and social activities: namely more social activities at $\mathrm{T} 1$ predict less depression at $\mathrm{T}_{2}$, and more depression at $\mathrm{T}_{2}$ implies fewer social activities at $\mathrm{T}_{3}$. In terms of the underlying psychological mechanisms that best describe the consequences of hearing loss, it was found that depression mediates the impact of hearing loss on reduced social activity. In other words, people who feel they have hearing problems may over time develop depression, which then may reduce their participation in social activities. The fact that the final model fits well for men and women, and across countries, shows the generalisation of these reciprocal causal effects. These results are in line with findings from Freedman et al. (2012), who found that, counter to their expectations, differences in participation did not account for disability's relationship with subjective wellbeing.

Although hearing loss is a common sensory deficit at older age, few studies can be found that focus on its specific consequences to emotional and social functioning. To our knowledge, this is the first study to test the unique role of perceived hearing loss and to address the processes through which it affects depression and social activity. This study found that hearing loss has the capacity to restrict older people's social activities, since it can generate depression. As social relationships and social support are important for health (Cohen 2004), hearing loss most likely motivates a cycle of increasingly disrupted communicative social and emotional interactions that make it hard to benefit from usual social support. As this study showed a reciprocal relationship between depression and social activities, this then may lead to reduced wellbeing and, again, to the disengagement from interpersonal relations and social activities.

Regardless of its silent impact, people tend to underestimate hearing loss and few seek medical help. Some common reasons that elderly people do not seek help are the lack of awareness of the problem and/or its consequences, or that that they perceive the symptoms, especially mild chronic ones, as part of the ageing process that cannot be helped (see e.g. Carson 2005; Ciorba et al. 2012). Also, another reason relates to the perceived 
stigma associated with hearing loss and the use of a hearing aid (St Claire and He 2009; Wallhagen 2009). Knowing the impact of hearing loss, and the mechanisms that explain these restrictions, may lead the development of public health detection and rehabilitation interventions that prevent the negative consequences of this sensory impairment. For example, increased depression symptoms may be one of the first signs that an older person is losing their hearing, which can help early diagnoses.

In fact, adequate and timely screening may be essential for the recovery of sensory function and to improve the communicational condition, but also to ameliorate mental health and quality of life, active ageing and social integration, which also contribute to a better overall state of health (Silva 2014). Interestingly, depression at $\mathrm{T}_{1}$ also predicted perceived hearing loss at T2, which shows that the perceptions of hearing loss may also be worsened by people's poor mental state, and that the greater the depression, the worse the perception of the hearing problems.

Despite contributing to the literature on the role of hearing loss in depression and social engagement, the present study has some limitations. First, as we used the databases from the SHARE, our analyses were based on the available questions for assessing perceived hearing loss, depression and social activities. For example, we could not use the SHARE's items 'caring for a sick or disabled adult' and 'provide help to family, friends or neighbours' to assess social activity (e.g. Viljanen et al. 2014) because these were only used in Waves 1 and 2, not Wave 4. Instead, we adapted and included a measure on 'looking after grandchildren' that was common across the three waves. A second limitation relates to the fact that hearing loss was assessed based solely on self-report. Although several researchers have pointed to the validity of self-reported health indicators (e.g. Idler and Benyamini 1997), objectively measured hearing loss would have strengthened the analyses and should be incorporated in future studies. Also, it is important to note that we did not consider whether participants did or did not use a hearing aid because our focus was on the perceived ability to hear, regardless of hearing aid usage (in fact, the SHARE question explicitly refers to that: 'Is your hearing (using a hearing aid as usual) excellent/ very good/good/fair/poor?'). To check whether there were overall differences on depression levels between participants who wore a hearing aid and those who did not, we compared the two groups in all waves, and no differences were found $\left(\operatorname{mean}_{\mathrm{T}_{1}}=2.21, \mathrm{SD}=2.00, \mathrm{~N}=392\right.$ versus mean $_{\mathrm{T}_{1}}$ $=2.20, \quad \mathrm{SD}=2.12, \mathrm{~N}=9,696 ; \operatorname{mean}_{\mathrm{T}_{2}}=2.23, \mathrm{SD}=2.00, \mathrm{~N}=491$ versus mean $_{\mathrm{T}_{2}}=2.14, \mathrm{SD}=2.09, \mathrm{~N}=9,597 ; \operatorname{mean}_{\mathrm{T}_{3}}=2.41, \mathrm{SD}=2.14, \mathrm{~N}=803$ versus mean $_{\mathrm{T}_{3}}=2.36, \mathrm{SD}=2.17, \mathrm{~N}=9,285$, respectively). However, it is important that future longitudinal studies investigate whether using a hearing aid can prevent or minimise the negative consequences of 
hearing loss in terms of depression and social activity (e.g. Mulrow, Tuley and Aguilar 1992).

Although we have used a cross-lagged longitudinal design that is robust to the effect of confounding variables by taking into account the auto-regressive effect, it does not overcome the limitations in the measurement of hearing loss, depression and social activities. In other words, it is important to consider that the depression and the less involvement in social activities resulting from hearing loss may also be due to other factors not controlled in this study, such as comorbidities or the decrease of physical or cognitive abilities. Finally, we did not have control of the temporal gap between the three waves, meaning it is possible that the two-year versus four-year gap may have influenced the results. Even if these limitations can mitigate the strength of conclusions that can be drawn from the results, the strengths of this study include the use of longitudinal data that involved a representative sample of elderly people from ten different countries.

The current research represents an important contribution to the study of hearing loss because it overcomes a limitation of previous cross-sectional studies by enabling us to examine the sequence of effects resulting from hearing loss at older age but more studies are needed to confirm these conclusions. The auto-regressive cross-lagged models allow not only verification of how much change across time in one construct is likely to be related to another construct in a temporal sequence but also allow attenuation of the confounding of other variables not present in the study design. In fact, one of the most important benefits of the cross-lagged longitudinal analysis is the opportunity to control for confounding third variables by taking into account prior levels of the dependent variable, being this control carried out in the auto-regressive effect (Cole and Maxwell 2003), because this effect minimizes the possibility of the cross-lagged effect be due to a third variable correlated with both the predictor and outcome variables.

Important possible confounding covariates are physical and cognitive functioning. In particular, although there is no clear evidence whether hearing loss is the cause of reduced cognitive performance or if both are parts of a common process, strong correlations have been found between hearing loss and loss of cognitive functions, independent of age and educational level (Arlinger 2003). More research is needed to clarify these relationships.

The perceived stigma associated with hearing loss may affect the initial acceptance of the disability and the rejection of hearing assessment and treatment (Wallhagen 2oog). This study has confirmed its impact on depression and consequent deterioration of social functioning. It will be of great importance to value hearing loss by enhancing methods of assessment and treatment, and by helping older adults to maintain self-esteem and participation in social situations. 


\section{Supplementary Material}

To view supplementary material for this article, please visit https://doi.org/ $10.1017 /{\text { So } 144686 X_{1} 7000708}^{2}$

\section{Acknowledgements}

This work, which was part of the wider research programme - 'Age-related Hearing Loss: Genetic Risk Factors and Social Impact' - was supported by the Portuguese Foundation for Science and Technology (PTDC/NEU-BEN/1192/2012). Preparation of the paper was also supported by two postdoctoral grants from the Portuguese Foundation for Science and Technology awarded to the first (SFRH/ $\left.\mathrm{BPD} / 983^{8} 5 / 2013\right)$ and third (SFRH/BPD/92242/2013) authors.

\section{References}

American Psychiatric Association 2013. Diagnostic and Statistical Manual of Mental Disorders. Fifth edition, American Psychiatric Publishing, Arlington, Virginia.

Arlinger, S. 2003. Negative consequences of uncorrected hearing loss - a review. International Journal of Audiology, 42, 2, S1 7-20.

Bess, F. H., Lichtenstein, M.J. and Logan, S. A. 1990. Making hearing impairment functionally relevant: linkages with hearing disability and handicap. Acta Oto-Laryngologica. Supplementum, 476, July, 226-31.

Bess, F. H., Lichtenstein, M.J., Logan, S. A, Burger, M. C. and Nelson, E. 1989. Hearing impairment as a determinant of function in the elderly. Journal of the American Geriatrics Society, 37, 2, 123-8.

Börsch-Supan, A. and Jürges, H. (eds) 2005. The Survey of Health, Ageing and Retirement in Europe: Methodology. Mannheim Research Institute for the Economics of Aging, Mannheim, Germany.

Byrne, B. 2009. Structural Equation Modeling with AMOS. Routledge, London.

Carabellese, C., Appollonio, I., Rozzini, R., Bianchetti, A., Frisoni, G. B., Frattola, L. and Trabucchi, M. 1993. Sensory impairment and quality of life in a community elderly population. Journal of the American Geriatrics Society, 41, 4, 401-7.

Carson, A. J. 2005. 'What brings you here today?' The role of self-assessment in helpseeking for age-related hearing loss. Journal of Aging Studies, 19, 2, $185^{-200 .}$

Chen, D. S., Genther, D. J., Betz, J. and Lin, F. R. 2014 . Association between hearing impairment and self-reported difficulty in physical functioning. Journal of the American Geriatrics Society, 62, 5, 850-6.

Chen, J., Liang, J., Ou, J. and Cai, W. 2013. Mental health in adults with sudden sensorineural hearing loss: an assessment of depressive symptoms and its correlates. Journal of Psychosomatic Research, 75, 1, 72-4.

Chou, K. L. 2008. Combined effect of vision and hearing impairment on depression in older adults: evidence from the English Longitudinal Study of Ageing. Journal of Affective Disorders, 106, 1/2, 191-6.

Chou, K.-L. and Chi, I. 2004. Combined effect of vision and hearing impairment on depression in elderly Chinese. International Journal of Geriatric Psychiatry, 19, $9,825^{-32 .}$ 
Ciorba, A., Bianchini, C., Pelucchi, S. and Pastore, A. 201 2. The impact of hearing loss on the quality of life of elderly adults. Clinical Interventions in Aging, 7, 6, 159-63.

Cohen, S. 2004. Social relationships and health. The American Psychologist, 59, 8, $676-84$.

Cole, D. A. and Maxwell, S. E. 2003. Testing mediational models with longitudinal data: questions and tips in the use of structural equation modeling. Journal of Abnormal Psychology, 112, 4, 55 $8-77$.

Cornwell, E.Y. and Waite, L.J. 2009. Social disconnectedness, perceived isolation, and health among older adults. Journal of Health and Social Behavior, 5o, 1, 31-48.

Crews, J. E. and Campbell, V. A. 2004. Vision impairment and hearing loss among community-dwelling older Americans: implications for health and functioning. American Journal of Public Health, 94, 5, 823-9.

Dalton, D. S., Cruickshanks, K.J., Klein, B. E. K., Klein, R., Wiley, T. L. and Nondahl, D. M. 2003. The impact of hearing loss on quality of life in older adults. The Gerontologist, 43, 5, 661-8.

Duncan, T. E., Duncan, S. C. and Stryker, L. A. 2006. An Introduction to Latent Variable Growth Curve Modeling: Concepts, Issues, and Applications. Second edition, Erlbaum, Mahwah, New Jersey.

Finkel, S. E. 1995. Causal Analysis with Panel Data. Sage, Thousand Oaks, CA.

Freedman, V.A., Stafford, F., Schwarz, N., Conrad, F. and Cornman, J. C. 2012. Disability, participation, and subjective wellbeing among older couples. Social Science and Medicine, 74, 4, 588-96.

Gana, K., Saada, Y., Broc, G., Quintard, B., Amieva, H. and Dartigues, J. F. 2016. As long as you've got your health: longitudinal relationships between positive affect and functional health in old age. Social Science and Medicine, 150, 231-8.

Gussekloo, J., De Bont, L. E., Von Faber, M., Eekhof, J. A., De Laat, J. A., Hulshof, J. H., Van Dongen, E. and Westendorp, R. G. 2003. Auditory rehabilitation of older people from the general population - the Leiden 85 -plus study. British Journal of General Practice, 53, 492, 536-4o.

Hallberg, L. R., Påsse, U. and Jansson, G. 1999. Quality of life among women with noise-induced hearing loss. Scandinavian Journal of Disability Research, 1, 1, 23-37.

Harada, S., Nishiwaki, Y., Michikawa, T., Kikuchi, Y., Iwasawa, S., Nakano, M., Ishigami, A., Saito, H. and Takebayashi, T. 2008. Gender difference in the relationships between vision and hearing impairments and negative well-being. Preventive Medicine, 47, 4, 433-7.

Heine, C. and Browning, C. J. 2004. The communication and psychosocial perceptions of older adults with sensory loss: a qualitative study. Ageing $\mathcal{E}^{2}$ Society, 24, 1 , $113-30$.

Helvik, A.-S., Jacobsen, G. and Hallberg, L. R.-M. 2006. Psychological well-being of adults with acquired hearing impairment. Disability and Rehabilitation, 28, 9, 535-45.

Idler, E. L. and Benyamini, Y. 1997. Self-rated health and mortality: a review of twenty-seven community studies. Journal of Health and Social Behavior, 38, 1, 21-37.

Jones, D. A., Victor, C. R. and Vetter, N. J. 1984. Hearing difficulty and its psychological implications for the elderly. Journal of Epidemiology and Community Health, 38, $1,75^{-8 .}$

Jürges, H. 2005. Interview, module, and question length in SHARE. In BörschSupan, A. and Jürges, H. (eds), The Survey of Health, Ageing and Retirement in Europe: Methodology. Mannheim Research Institute for the Economics of Aging, Mannheim, Germany, 82-7.

Millán-Calenti, J. G., Maseda, A., Rochette, S. and García-Monasterio, I. 2011. Relationship between sensory hearing loss and depression in elderly people: a literature review. Revista Espanola de Geriatria y Gerontologia, 46, 1, 30-5. 
Mulrow, C. D., Tuley, M. R. and Aguilar, C. 1992. Sustained benefits of hearing aids. Journal of Speech, Language, and Hearing Research, 35, 6, 1402-5.

Prince, M. J., Reischies, F., Beekman, A. T., Fuhrer, R., Jonker, C., Kivela, S. L., Lawlor, B. A., Lobo, A., Magnusson, H., Fichter, M., van Oyen, H., Roelands, M., Skoog, I., Turrina, C. and Copeland, J. R. 1999. Development of the EURO-D scale - a European Union initiative to compare symptoms of depression in 14 European centres. British Journal of Psychiatry, 174, 330-8.

Schumacker, R. E. and Lomax, R. G. 1996. A Beginner's Guide to Structural Equation Modeling. Lawrence Erlbaum Associates, Mahwah, New Jersey.

Selig, J. P. and Little, T. D. 2012. Autoregressive and cross-lagged panel analysis for longitudinal data. In Laursen, B., Little, T. D. and Card, N. (eds), Handbook of Developmental Research Methods. Guilford Press, New York, $265^{-} 78$.

Silva, P.A. 2014. Social and individual determinants of subjective health status and well-being of the senior population. Cadernos de Saúde Pública [Reports in Public Health], 3o, $11,2387-400$.

Silverstein, M. and Parker, M. G. 2002. Leisure activities and quality of life among the oldest old in Sweden. Research on Aging, 24, 5, 528-47.

St Claire, L. S. and He, Y. 2009. How do I know if I need a hearing aid? Further support for the self-categorisation approach to symptom perception. Applied Psychology, 58, 1, 24-41.

Stephens, S. D. 1980. Evaluating the problems of the hearing impaired. Audiology: Official Organ of the International Society of Audiology, 19, 3, $205^{-20 .}$

Viljanen, A., Törmäkangas, T., Vestergaard, S. and Andersen-Ranberg, K. 2014. Dual sensory loss and social participation in older Europeans. European Journal of Ageing, $\mathbf{1 1}, 2,155^{-67}$.

Wallhagen, M. I. 2009. The stigma of hearing loss. Gerontologist, 5o, 1, 66-75.

Wallhagen, M. I., Strawbridge, W.J., Shema, S. J., Kurata, J. and Kaplan, G. A. 2001. Comparative impact of hearing and vision impairment on subsequent functioning. Journal of the American Geriatrics Society, 49, 8, 1086-92.

Wilkie, R., Peat, G., Thomas, E. and Croft, P. 2007. Factors associated with participation restriction in community-dwelling adults aged 5 o years and over. Quality of Life Research, 16, 7, $1147-56$.

Yamada, M., Nishiwaki, Y., Michikawa, T. and Takebayashi, T. 2012. Self-reported hearing loss in older adults is associated with future decline in instrumental activities of daily living but not in social participation. Journal of the American Geriatrics Society, 6o, 7, 1304-9.

Accepted I 4 June 2017

Address for correspondence:

Cláudia Campos Andrade,

CIS-IUL, ISCTE-IUL (Instituto Universitário de Lisboa), Av. das Forças Armadas, 1649-026 Lisboa, Portugal

E-mail: claudia.andrade@iscte.pt 